\title{
An Analytic Form for the Effective Lagrangian of QED and its Application to Pair Production and Photon Splitting
}

\author{
Jeremy S. Heyl, Lars Hernquist* \\ Lick Observatory, University of California, Santa Cruz, California 95064, USA
}

\begin{abstract}
We derive an analytic form for the Heisenberg-Euler Lagrangian in the limit where the component of the electric field parallel to the magnetic field is small. We expand these analytic functions to all orders in the field strength $\left(F_{\mu \nu} F^{\mu \nu}\right)$ in the limits of weak and strong fields, and use these functions to estimate the pair-production rate in arbitrarily strong electric fields and the photon-splitting rate in arbitrarily strong magnetic fields.
\end{abstract}

12.20.Ds, 97.60.Jd, 98.70.Rz

\section{INTRODUCTION: THE ONE-LOOP EFFECTIVE LAGRANGIAN OF QED}

When one-loop corrections are included in the Lagrangian of the electromagnetic field one obtains a non-linear correction term:

$$
\mathcal{L}=\mathcal{L}_{0}+\mathcal{L}_{1}
$$

Both terms of the Lagrangian can be written in terms of the Lorentz invariants,

$$
I=F_{\mu \nu} F^{\mu \nu}=2\left(|\vec{B}|^{2}-|\vec{E}|^{2}\right)
$$

and

$$
K=\left\{\epsilon^{\lambda \rho \mu \nu} F_{\lambda \rho} F_{\mu \nu}\right\}^{2}=-(4 \vec{E} \cdot \vec{B})^{2},
$$

following Heisenberg and Euler [1]. We do not expect terms which are odd powers of $\epsilon^{\lambda \rho \mu \nu} F_{\lambda \rho} F_{\mu \nu}$ to appear in the effective Lagrangian as these terms would yield a Lagrangian which would violate the $C$ and $P$ symmetries of the tree-level Lagrangian.

Heisenberg and Euler [1] and Weisskopf [2] independently derived the effective Lagrangian of the electromagnetic field using electron-hole theory. Schwinger 33 later rederived the same result using quantum electrodynamics. In rationalized electromagnetic units, the Lagrangian is given by

$$
\begin{aligned}
& \mathcal{L}_{0}=-\frac{1}{4} I \\
& \mathcal{L}_{1}=\frac{e^{2}}{h c} \int_{0}^{\infty} e^{-\zeta} \frac{\mathrm{d} \zeta}{\zeta^{3}}\left\{i \zeta^{2} \frac{\sqrt{-K}}{4} \times\right. \\
& \left.\frac{\cos \left(\frac{\zeta}{B_{k}} \sqrt{-\frac{I}{2}+i \frac{\sqrt{-K}}{2}}\right)+\cos \left(\frac{\zeta}{B_{k}} \sqrt{-\frac{I}{2}-i \frac{\sqrt{-K}}{2}}\right)}{\cos \left(\frac{\zeta}{B_{k}} \sqrt{-\frac{I}{2}+i \frac{\sqrt{-K}}{2}}\right)-\cos \left(\frac{\zeta}{B_{k}} \sqrt{-\frac{I}{2}-i \frac{\sqrt{-K}}{2}}\right)}+\left|B_{k}\right|^{2}+\frac{\zeta^{2}}{6} I\right\} .
\end{aligned}
$$

where $B_{k}=E_{k}=\frac{m^{2} c^{3}}{e \hbar} \approx 2.2 \times 10^{15} \mathrm{~V} \mathrm{~cm}^{-1} \approx 4.4 \times 10^{13} \mathrm{G}$. Both Heisenberg and Euler [1], and Mielniczuk [4] present alternative expressions for these integrals in terms of infinite series.

${ }^{*}$ Presidential Faculty Fellow 


\section{THE ANALYTIC EXPANSION}

For many interesting problems, one needs an expansion of this Lagrangian in the limit where the component of the electric field in the direction of the magnetic field is small (small $K$ )

$$
\mathcal{L}_{1}=\mathcal{L}_{1}(I, 0)+\left.K \frac{\partial \mathcal{L}_{1}}{\partial K}\right|_{K=0}+\left.\frac{K^{2}}{2} \frac{\partial^{2} \mathcal{L}_{1}}{\partial K^{2}}\right|_{K=0}+\cdots
$$

where the terms are given by the following integrals

$$
\begin{aligned}
\mathcal{L}_{1}(I, 0)= & \frac{e^{2}}{h c} \frac{I}{2} X_{0}\left(\frac{1}{\xi}\right) \\
= & \frac{e^{2}}{h c} \frac{I}{2} \int_{0}^{\infty} e^{-u / \xi} \frac{\mathrm{d} u}{u^{3}}\left(-u \operatorname{coth} u+1+\frac{u^{2}}{3}\right) \\
\left.\frac{\partial \mathcal{L}_{1}}{\partial K}\right|_{K=0}= & \frac{e^{2}}{h c} \frac{1}{16 I} X_{1}\left(\frac{1}{\xi}\right) \\
= & \frac{e^{2}}{h c} \frac{1}{16 I} \int_{0}^{\infty} e^{-u / \xi} \frac{\mathrm{d} u}{u^{2}}\left(\operatorname{coth} u-\frac{2}{3} u^{2} \operatorname{coth} u-u \operatorname{csch}^{2} u\right) \\
\left.\frac{\partial^{2} \mathcal{L}_{1}}{\partial K^{2}}\right|_{K=0}= & \frac{e^{2}}{h c} \frac{1}{384 I^{3}} X_{2}\left(\frac{1}{\xi}\right) \\
= & \frac{e^{2}}{h c} \frac{1}{384 I^{3}} \int_{0}^{\infty} e^{-u / \xi} \frac{\mathrm{d} u}{u^{2}}\left(-9 u \operatorname{csch}^{2} u-4 u^{3} \operatorname{csch}^{2} u\right. \\
& \left.+2 u^{2} \operatorname{coth} u+15 \operatorname{coth} u+\frac{8}{15} u^{4} \operatorname{coth} u-6 u^{2} \operatorname{coth}^{3} u\right)
\end{aligned}
$$

and we have defined

$$
\xi=\frac{1}{B_{k}} \sqrt{\frac{I}{2}} \text { and } u=\frac{\zeta}{B_{k}} \sqrt{\frac{I}{2}} .
$$

Note that $\xi$ is a dimensionless measure of the strength of the field.

The auxiliary functions $X_{i}$ may be calculated analyically:

$$
\begin{aligned}
X_{0}(x)= & \int_{0}^{x / 2-1} \ln (\Gamma(v+1)) \mathrm{d} v+\frac{1}{3} \ln \left(\frac{1}{x}\right)+2 \ln 4 \pi-4 \ln A-\frac{5}{3} \ln 2 \\
& -\left[\ln 4 \pi+1+\ln \left(\frac{1}{x}\right)\right] x+\left[\frac{3}{4}+\frac{1}{2} \ln \left(\frac{2}{x}\right)\right] x^{2} \\
X_{1}(x)=- & 2 X_{0}(x)+x X_{0}^{(1)}(x)+\frac{2}{3} X_{0}^{(2)}(x)-\frac{2}{9} \frac{1}{x^{2}} \\
X_{2}(x)=- & 24 X_{0}(x)+9 x X_{0}^{(1)}(x)+\left(8+3 x^{2}\right) X_{0}^{(2)}(x)+4 x X_{0}^{(3)}(x) \\
& -\frac{8}{15} X_{0}^{(4)}(x)+\frac{8}{15} \frac{1}{x^{2}}+\frac{16}{15} \frac{1}{x^{4}}
\end{aligned}
$$

where

$$
X_{0}^{(n)}(x)=\frac{\mathrm{d}^{n} X_{0}(x)}{\mathrm{d} x^{n}}
$$

Because of the near symmetry between $I$ and $K$ in Eq. 6, higher derivatives with respect to $K$ may be calculated in principle, and represented by a sum of derivatives of $\mathcal{L}(I, 0)$ with respect to $I$. The constant $A$ is defined as

$$
\begin{aligned}
\ln A & =\lim _{n \rightarrow \infty}\left(\sum_{i=1}^{n} i \ln i\right)-\left[\left(\frac{n^{2}}{2}+\frac{n}{2}+\frac{1}{12}\right) \ln n-\frac{n^{2}}{4}\right] \\
& =\frac{1}{12}-\zeta^{(1)}(-1)=0.248754477
\end{aligned}
$$


in analogy to the Euler-Mascheroni constant [5]. Here $\zeta^{(1)}(x)$ denotes the first derivative of the Riemann Zeta function.

Barnes [5] evaluates the definite integral of $\ln \Gamma(x)$ in terms of the $G$-function

$$
\int_{0}^{x / 2-1} \ln (\Gamma(v+1)) \mathrm{d} v=\left(\frac{x}{2}-1\right) \ln \Gamma\left(\frac{x}{2}\right)-\ln G\left(\frac{x}{2}\right)-\frac{x^{2}}{8}+\frac{x}{4}(1+\ln 2 \pi)-\frac{1}{2} \ln 2 \pi .
$$

where

$$
G(z)=(2 \pi)^{\frac{z-1}{2}} e^{-\frac{z(z-1)}{2}} e^{-\gamma \frac{(z-1)^{2}}{2}} \prod_{k=1}^{\infty}\left[\left(1+\frac{z-1}{k}\right)^{k} e^{1-z+\frac{(z-1)^{2}}{2 k}}\right] .
$$

The integral of $\ln \Gamma(x)$ may also be expressed in terms of the generalized Riemann Zeta function [6]

$$
\begin{aligned}
\int_{0}^{x / 2-1} \ln (\Gamma(v+1)) \mathrm{d} v=\zeta^{(1)} & \left(-1, \frac{x}{2}-1\right)-\zeta^{(1)}(-1)+\frac{\ln 2 \pi}{2}\left(\frac{x}{2}-1\right) \\
& -\frac{x}{4}\left(\frac{x}{2}-1\right)+\left(\frac{x}{2}-1\right) \ln \left(\frac{x}{2}-1\right)
\end{aligned}
$$

Our expression for $X_{0}$ was also found by Dittrich et al. [6]. Ivanov [7] derived a similar expression as well, but his result differs from ours and that of Dittrich et al. in the constant term. Unlike Ivanov's expression, ours approaches zero as $\xi$ goes to 0 which from examination of Eq. 7 is the correct limiting behavior. In addition, the above form for $X_{0}$ reproduces the asymptotic strong-field limit given by Heisenberg and Euler [1].

These functions can be expanded in both the weak-field and strong-field limits. In the weak-field limit $(\xi<0.5)$ we obtain

$$
\begin{aligned}
& X_{0}\left(\frac{1}{\xi}\right)=-\sum_{j=1}^{\infty} \frac{2^{2 j} B_{2(j+1)}}{j(j+1)(2 j+1)} \xi^{2 j} \\
& X_{1}\left(\frac{1}{\xi}\right)=-\frac{14}{45} \xi^{2}+\frac{1}{3} \sum_{j=2}^{\infty} \frac{2^{2 j}\left(6 B_{2(j+1)}-(2 j+1) B_{2 j}\right)}{j(2 j+1)} \xi^{2 j} \\
& X_{2}\left(\frac{1}{\xi}\right)=\frac{1}{15} \sum_{j=3}^{\infty} \frac{2^{2 j}}{j}\left[2 j(2 j-1) B_{2(j-1)}+60(j-1) B_{2 j}\right. \\
&\left.-180 \frac{j-2}{2 j+1} B_{2(j+1)}\right] \xi^{2 j}
\end{aligned}
$$

where $B_{j}$ denotes the $j$ th Bernoulli number. In the strong-field limit $(\xi>0.5)$, we obtain

$$
\begin{aligned}
X_{0}\left(\frac{1}{\xi}\right)=( & \left.\frac{1}{3} \ln 2-4 \ln A+\frac{1}{3} \ln \xi\right)+(1-\ln \pi+\ln \xi) \xi^{-1} \\
& +\left(\frac{1}{2}(\ln 2-\gamma)+\frac{3}{4}+\frac{1}{2} \ln \xi\right) \xi^{-2} \\
& +\sum_{j=3}^{\infty} \frac{(-1)^{j-1}}{2^{j-2}} \frac{1}{j} \frac{1}{j-1} \zeta(j-1) \xi^{-j} \\
X_{1}\left(\frac{1}{\xi}\right)=- & \frac{2}{3} \xi+\left(8 \ln A-\frac{1}{3}-\frac{2}{3} \gamma\right) \\
& +\left(\ln \pi+\frac{1}{18} \pi^{2}-2-\ln \xi\right) \xi^{-1}+\left(-\frac{1}{2}-\frac{1}{6} \zeta(3)\right) \xi^{-2} \\
& +\sum_{j=3}^{\infty} \frac{(-1)^{j-1}}{2^{j-2}}\left(\frac{j-2}{j(j-1)} \zeta(j-1)+\frac{1}{6} \zeta(j+1)\right) \xi^{-j}
\end{aligned}
$$




$$
\begin{aligned}
X_{2}\left(\frac{1}{\xi}\right)=\frac{16}{15} & \xi^{3}-4 \xi+\left(-6-8 \gamma+\frac{4}{15} \zeta(3)+96 \ln A\right) \\
+ & \left(-27+\pi^{2}-15 \ln \pi-\frac{1}{225} \pi^{4}-15 \ln \xi\right) \xi^{-1} \\
+ & \left(\frac{2}{5} \zeta(5)-9-4 \zeta(3)\right) \xi^{-2} \\
+ & \sum_{j=3}^{\infty} \frac{(-1)^{j-1}}{2^{j-2}}\left[\frac{3(j+4)(j-2)}{j(j-1)} \zeta(j-1)\right. \\
& \left.+(j+2)\left(\zeta(j+1)-\frac{j+1}{30} \zeta(j+3)\right)\right] \xi^{-j}
\end{aligned}
$$

The Lagrangian may also be expanded in terms of the invariants themselves or the electric and magnetic fields. In the weak-field limit we obtain

$$
\begin{aligned}
\mathcal{L}_{1}(I, 0) & =\frac{e^{2}}{h c}\left(\frac{1}{180} \frac{I^{2}}{B_{k}^{2}}-\frac{1}{630} \frac{I^{3}}{B_{k}^{4}}+\frac{1}{630} \frac{I^{4}}{B_{k}^{6}}+\cdots\right) \\
\left.\frac{\partial \mathcal{L}_{1}}{\partial K}\right|_{K=0} & =\frac{e^{2}}{h c}\left(-\frac{7}{720} \frac{1}{B_{k}^{2}}+\frac{13}{5040} \frac{I}{B_{k}^{4}}-\frac{11}{3780} \frac{I}{B_{k}^{6}}+\cdots\right) \\
\left.\frac{\partial^{2} \mathcal{L}_{1}}{\partial K^{2}}\right|_{K=0} & =\frac{e^{2}}{h c}\left(\frac{19}{15120} \frac{1}{B_{k}^{6}}-\frac{127}{23760} \frac{I}{B_{k}^{8}}+\frac{5527}{180180} \frac{I^{2}}{B_{k}^{10}}+\cdots\right) .
\end{aligned}
$$

This weak-field expansion agrees with the Heisenberg and Euler [1] result.

In the strong-field limit, for direct comparison with Heisenberg and Euler [1] we define $a=E / E_{k}$ and $b=B / B_{k}$ and take the limit $b \gg 1$ and $a \ll 1$. We take, $\xi^{2}=b^{2}-a^{2}, \xi \approx b-\frac{a^{2}}{2 b}$ and $K=-16 B_{k}^{4}(a b)^{2}$. We obtain

$$
\begin{aligned}
\mathcal{L}_{1}(a, b)=4 \frac{e^{2}}{h c} & B_{k}^{2}\left[b^{2}\left(\frac{\ln b}{12}-\ln A+\frac{\ln 2}{12}\right)+\frac{b}{4}(\ln b+1-\ln \pi)\right. \\
& +\frac{\ln b}{8}+\frac{3}{16}+\frac{\ln 2-\gamma}{8} \\
& -\frac{a^{2}}{12}(\ln b+\ln 2-\gamma) \\
& \left.+b\left(\frac{a^{2}}{12}+\frac{a^{4}}{90}+\cdots\right)+\cdots\right]
\end{aligned}
$$

which agrees numerically with the corresponding expansion in Heisenberg and Euler [1].

\section{PAIR PRODUCTION IN AN ARBITRARILY STRONG ELECTRIC FIELD}

In a strong electric field with no magnetic field, the value of the first invariant is negative, $I=-2|\vec{E}|^{2}$ and $K=0$.

The analytic expressions for the Lagrangian are valid for values of $\xi$ throughout the complex plane, with a branch cut along the negative real axis. Using an imaginary value of

$$
\xi=i\left(\frac{E}{E_{k}}\right)=i y, y>0
$$

and taking $w=2(4 \pi \hbar)^{-1} \operatorname{Im} \mathcal{L}$ gives the pair production rate per unit volume 8 . From examination of Eq. 20, for $\xi<0.5$ the pair-production rate is apparently zero. However, since Eq. 20 is a power series in $\xi / 2$, the imaginary part of $X_{0}$ may be exponentially small. Berestetskii et al. [8] derive for a weak field,

$$
w=2(4 \pi \hbar)^{-1} \operatorname{Im} \mathcal{L} \sim \frac{1}{4 \pi^{3}}\left(\frac{\hbar}{m c}\right)^{-3}\left(\frac{\hbar}{m c^{2}}\right)^{-1} y^{2} \exp \left(-\frac{\pi}{y}\right)
$$


To simplify the numerics we use an alternate definition of $X_{0}(x)$ obtained by means of a change of variables

$$
\begin{aligned}
X_{0}(x)=4 & \left(\frac{x}{2}-1\right) \int_{0}^{1} \ln \left(\Gamma\left(u\left(\frac{x}{2}-1\right)+1\right)\right) \mathrm{d} u+\frac{1}{3} \ln \left(\frac{1}{x}\right) \\
& +2 \ln 4 \pi-\left(4 \ln A+\frac{5}{3} \ln 2\right)-\left[\ln 4 \pi+1+\ln \left(\frac{1}{x}\right)\right] x \\
& +\left[\frac{3}{4}+\frac{1}{2} \ln \left(\frac{2}{x}\right)\right] x^{2} .
\end{aligned}
$$

With this definition and the property of the Gamma function, $\ln \Gamma(\bar{x})=\overline{\ln \Gamma(x)}$, we see that $X_{0}(\bar{x})=\overline{X_{0}(x)}$, so

$$
\begin{aligned}
w= & \left.2(4 \pi \hbar)^{-1} \operatorname{Im} \mathcal{L}\right|_{I=-2 y^{2} E_{k}^{2}, K=0} \\
= & i \frac{e^{2}}{8 \pi^{2} \hbar^{2} c} E_{k}^{2} y^{2}\left(X_{0}\left(-\frac{i}{y}\right)-X_{0}\left(\frac{i}{y}\right)\right) \\
= & \frac{1}{8 \pi^{2}}\left(\frac{\hbar}{m c}\right)^{-3}\left(\frac{\hbar}{m c^{2}}\right)^{-1}\left[-\frac{1}{3} \pi y^{2}-8 y^{2} \operatorname{Im} Q(y)\right. \\
& \left.\quad-2 y(\ln y+\ln 4 \pi+1)+4 y \operatorname{Re} Q(y)+\frac{1}{2} \pi\right]
\end{aligned}
$$

where

$$
Q(y)=\int_{0}^{1} \ln \left(\Gamma\left(u\left(\frac{i}{2 y}-1\right)+1\right)\right) \mathrm{d} u
$$

and the scaling constant

$$
\frac{1}{8 \pi^{2}}\left(\frac{\hbar}{m c}\right)^{-3}\left(\frac{\hbar}{m c^{2}}\right)^{-1}=1.7 \times 10^{51} \mathrm{~cm}^{-3} \mathrm{~s}^{-1}
$$

This expression for $w$, the pair-production rate, agrees numerically with Itzykson and Zuber's results [9] for an arbitrarily strong electric field.

In the strong-field limit we use Eq. 23 and take the imaginary part

$$
\begin{gathered}
w=\frac{1}{8 \pi^{2}}\left(\frac{\hbar}{m c}\right)^{-3}\left(\frac{\hbar}{m c^{2}}\right)^{-1}\left[-\frac{\pi}{3} y^{2}+2(1-\ln \pi+\ln y) y\right. \\
+\frac{\pi}{2}+\sum_{k=1}^{\infty} \frac{(-1)^{3 k} \zeta(2 k)}{2^{2 k-1} k(2 k+1)} y^{-(2 k-1)}
\end{gathered}
$$




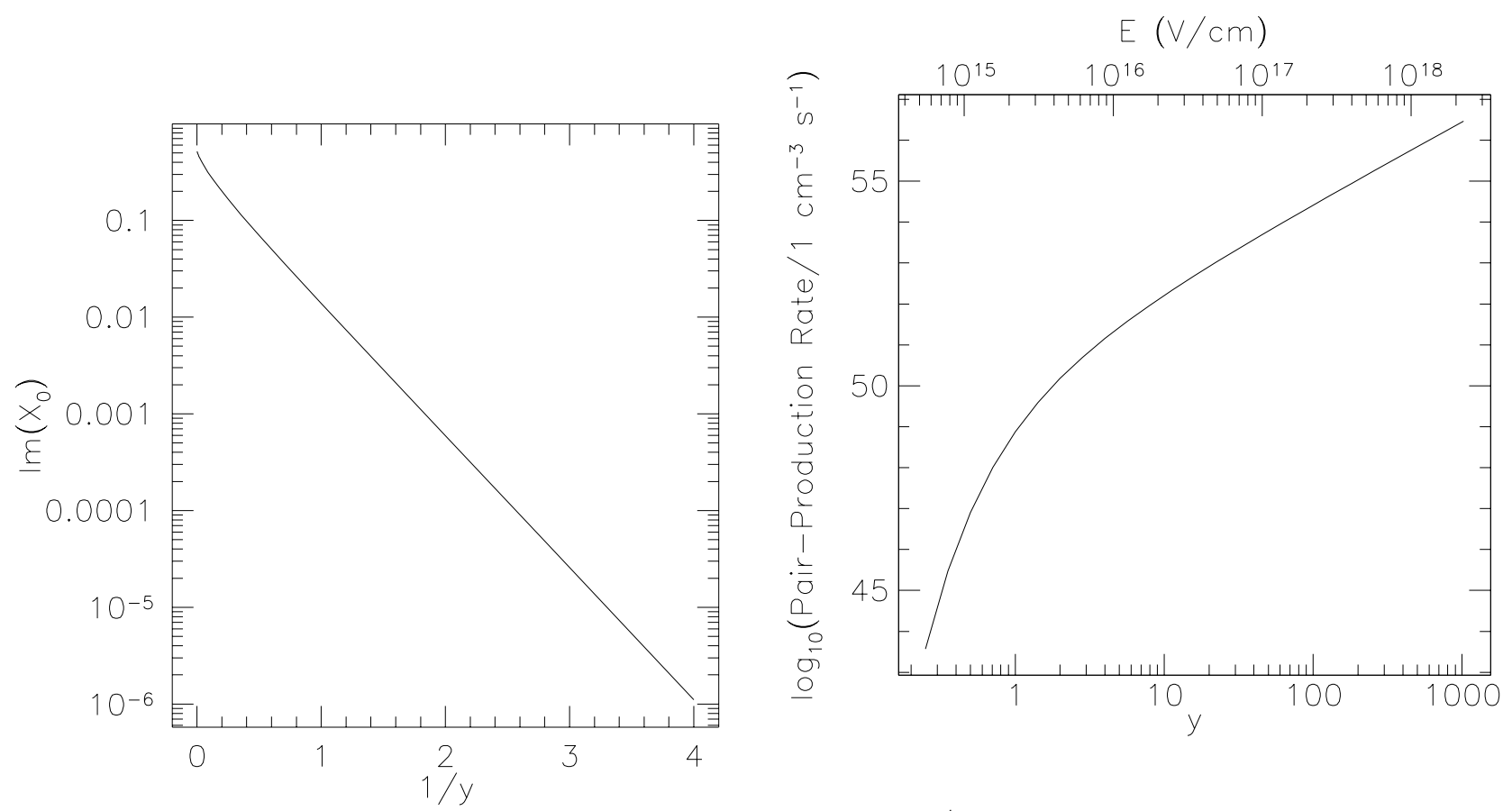

FIG. 1. The left panel depicts the imaginary part of $X_{0}$ as a function of $1 / y$. For weak fields the imaginary component is approximately $\pi^{-1} \exp (-\pi / y)$. The right panel depicts the pair-production rate for near and super-critical fields.

Fig. 11 depicts the imaginary component of $X_{0}$ for $I=-2 y^{2} E_{k}^{2}$ and the pair-production rate per unit volume. From the left panel, we verify that the imaginary component of $X_{0}$ is approximately $\pi^{-1} \exp (-\pi / y)$ for weak fields. The right panel shows the pair-production rate which increases as $y^{2}$ for strong fields and is damped exponentially in weak fields.

\section{AUXILIARY FUNCTIONS FOR PHOTON SPLITTING}

To calculate the photon splitting rates we follow the technique by Adler [10] for the low-frequency limit. In this limit, Adler expresses the opacity for photon spliting by means of auxiliary functions which are simply derivatives of the Lagrangian and therefore of the functions $X_{i}$ above

$$
\begin{aligned}
\kappa[\|\rightarrow\|+\|] & =\frac{\alpha^{6}}{2 \pi^{2}} \frac{\hbar^{13}}{m^{16} c^{24}} B^{6} \sin ^{6} \theta \frac{\omega^{5}}{30} C_{1}(\xi)^{2} \\
& =\frac{\alpha^{3}}{2 \pi^{2}}\left(\frac{B \sin \theta}{B_{k}}\right)^{6}\left(\frac{\hbar \omega}{m c^{2}}\right)^{5} \frac{C_{1}(\xi)^{2}}{30}\left(\frac{m c}{\hbar}\right) \\
& =17.0 \mathrm{~cm}^{-1}\left(\frac{B \sin \theta}{B_{k}}\right)^{6}\left(\frac{\hbar \omega}{m c^{2}}\right)^{5} C_{1}(\xi)^{2} \\
\kappa[\| \rightarrow \perp+\perp] & =\frac{\alpha^{6}}{2 \pi^{2}} \frac{\hbar^{13}}{m^{16} c^{24}} B^{6} \sin ^{6} \theta \frac{\omega^{5}}{30} C_{2}(\xi)^{2} \\
\kappa[\perp \rightarrow \|+\perp] & =2 \frac{\alpha^{6}}{2 \pi^{2}} \frac{\hbar^{13}}{m^{16} c^{24}} B^{6} \sin ^{6} \theta \frac{\omega^{5}}{30} C_{2}(\xi)^{2},
\end{aligned}
$$

The conversion of $\perp$ to $\|+\perp$ proceeds through two channels hence the two-fold increase in the opacity for this process. $C_{1}$ and $C_{2}$ are defined by

$$
\begin{aligned}
\left.\frac{\partial^{3} \mathcal{L}_{\text {Adler }}}{\partial \mathcal{F}^{3}}\right|_{\mathcal{G}=0, \mathcal{F}=\frac{1}{2} B^{2}} & =\left.\frac{64}{4 \pi} \frac{\partial^{3} \mathcal{L}_{1}}{\partial I^{3}}\right|_{K=0, I=2 B^{2}} \\
& =-\frac{\alpha^{3} \hbar^{6}}{2 \pi^{2} m^{8} c^{10}} C_{1}(\xi)=-\frac{\alpha}{2 \pi^{2} B_{k}^{4}} C_{1}(\xi)
\end{aligned}
$$




$$
\begin{aligned}
\left.\frac{\partial^{3} \mathcal{L}_{\text {Adler }}}{\partial \mathcal{F} \partial \mathcal{G}^{2}}\right|_{\mathcal{G}=0, \mathcal{F}=\frac{1}{2} B^{2}} & =-\left.\frac{128}{4 \pi} \frac{\partial^{2} \mathcal{L}_{1}}{\partial I \partial K}\right|_{K=0, I=2 B^{2}} \\
& =-\frac{\alpha}{2 \pi^{2} B_{k}^{4}} C_{2}(\xi)
\end{aligned}
$$

and $\theta$ is the angle between the direction of propagation of the photon and the external magnetic field. The factors of 128 and 64 result from the definitions of Adler's $\mathcal{F}$ and $\mathcal{G}$ in terms of $I$ and $K$,

$$
\begin{aligned}
\mathcal{F} & =\frac{1}{2}\left(|\vec{B}|^{2}-|\vec{E}|^{2}\right)=\frac{I}{4} \\
\mathcal{G} & =\vec{B} \cdot \vec{E}=\frac{1}{4} \sqrt{-K}
\end{aligned}
$$

An additional factor of $4 \pi$ appears because we are using rationalized Gaussian units while Adler employs unrationalized units.

Given the analytic forms for $X_{0}$ and $X_{1}$ we obtain

$$
\begin{aligned}
& C_{1}(\xi)=\frac{1}{4 \xi^{7}}\left(X_{0}^{(3)}\left(\frac{1}{\xi}\right)+3 X_{0}^{(2)}\left(\frac{1}{\xi}\right) \xi-3 X_{0}^{(1)}\left(\frac{1}{\xi}\right) \xi^{2}\right) \\
& C_{2}(\xi)=-\frac{1}{4 \xi^{5}}\left(X_{1}^{(1)}\left(\frac{1}{\xi}\right)+2 X_{1}\left(\frac{1}{\xi}\right) \xi\right)
\end{aligned}
$$

The functions, $C_{1}$ and $C_{2}$, have the appropriate limits as $\xi \rightarrow 0$ which correspond to the lowest order hexagon diagrams for the splitting process,

$$
\begin{aligned}
C_{1}(I) & =16 \sum_{j=2}^{\infty} \frac{2^{j-1}(j-1) B_{2(j+1)}}{2 j+1}\left(\frac{I}{B_{k}^{2}}\right)^{j-2} \\
& =6 \cdot \frac{8}{315}-\frac{64}{105} \frac{I}{B_{k}^{2}}+\frac{320}{99}\left(\frac{I}{B_{k}^{2}}\right)^{2} \cdots \\
C_{2}(I) & =\frac{1}{3} \sum_{j=2}^{\infty} \frac{2^{j+1}(j-1)\left(6 B_{2(j+1)}-(2 j+1) B_{2 j}\right)}{j(2 j+1)}\left(\frac{I}{B_{k}^{2}}\right)^{j-2} \\
& =6 \cdot \frac{13}{945}-\frac{176}{945} \frac{I}{B_{k}^{2}}+\frac{332}{495}\left(\frac{I}{B_{k}^{2}}\right)^{2} \cdots
\end{aligned}
$$

In the strong field limit we obtain

$$
\begin{aligned}
& C_{1}(\xi)=\frac{1}{3} \xi^{-4}-\frac{3}{4} \xi^{-5}\left(\ln \xi-\ln \pi+\frac{2}{3}\right)-\xi^{-6} \\
& \quad-\sum_{j=3}^{\infty} \frac{(-1)^{j}}{2^{j}} \frac{j^{2}-4}{j-1} \zeta(j-1) \xi^{-j-4} \\
& C_{2}(\xi)=\frac{1}{6} \xi^{-3}+\xi^{-4}\left(\frac{1}{6}+\frac{1}{3} \gamma-4 \ln A\right) \\
& \quad+\frac{3}{4} \xi^{-5}\left(\ln \xi-\ln \pi+\frac{5}{3}-\frac{1}{18} \pi^{2}\right)+\frac{1}{2} \xi^{-6}\left(1+\frac{1}{3} \zeta(3)\right) \\
& \quad+\sum_{j=3}^{\infty} \frac{(-1)^{j}(j+2)}{2^{j}}\left(\frac{j-2}{j(j-1)} \zeta(j-1)+\frac{\zeta(j+1)}{6}\right) \xi^{-j-4}
\end{aligned}
$$

These expressions for the photon-splitting rate are only valid in the low-frequency limit since the Heisenberg-Euler Lagrangian neglects the gradients of the field. When these gradients are neglected, the results from Schwinger's proper-time integration [3] used by Adler [10] reduce to these results obtained from the Heisenberg-Euler Lagrangian. Baǐer, Milstein and Shaisultanov [1] have also obtained similar results for arbitrary field strengths (and photon frequencies) using an operator diagram technique. 
Bă̌er et al. and Adler's methods differ in spirit but yield the same results. Bă̌r, Katkov, and Strakhovenko developed the operator diagram technique 12]. In this formalism, the photon splitting matrix element is evaluated with Feynman diagrams [13] using electron propagators in an external field. On the other hand, Adler [10] calculates the expectation value of the current $\left(\left\langle j_{\mu}(x)\right\rangle\right)$ order by order in the external photon fields using Schwinger's formalism [3] and relates this expectation value to the photon-splitting matrix element.

\section{PHOTON SPLITTING OPACITIES AND APPLICATION TO NEUTRON STARS}

Adler [10] argues that because of dispersive effects, the process $\| \rightarrow \perp+\perp$, dominates the opacity of photons travelling through a strong field. Therefore, we are interested in the function $C_{2}(\xi)$ which determines the splitting rate for all magnetic field strengths at photon energies small compared to the mass of the electron. We see immediately from the expansions of $C_{2}$ that the opacity has the following behavior for weak and strong fields

$$
\kappa[\| \rightarrow++\perp]= \begin{cases}0.116 \mathrm{~cm}^{-1}\left(\frac{B \sin \theta}{B_{k}}\right)^{6}\left(\frac{\hbar \omega}{m c^{2}}\right)^{5} & B \ll B_{k} \\ 0.472 \mathrm{~cm}^{-1} \sin ^{6} \theta\left(\frac{\hbar \omega}{m c^{2}}\right)^{5} & B \gg B_{k}\end{cases}
$$

We find, in agreement with the recent result of Baĭer et al. [11] and as well as with earlier results [14, 15] that the photon splitting opacity approaches a constant value in the limit of strong fields.

The left panel of Fig. 2 depicts the opacity for photons with $E=m c^{2}$ as a function of $\xi$. Our formulae are not valid for these high-energy photons but for low energies the opacity scales as this quantity times the photon energy to the fifth power. The right panel applies these opacities to neutron stars. Neutron stars are observed to have magnetic fields $\sim 10^{12} \mathrm{G}$ (e.g. $[16$ ) and a subset of these objects known as magnetars are suspected to have much stronger fields $\sim 10^{16} \mathrm{G}$ or larger 17. The figure illustrates the energy of photons with a mean-free path of ten kilometers. All parallel-polarized photons with this energy or larger would tranverse an optical depth of one or larger while escaping from the neutron star.
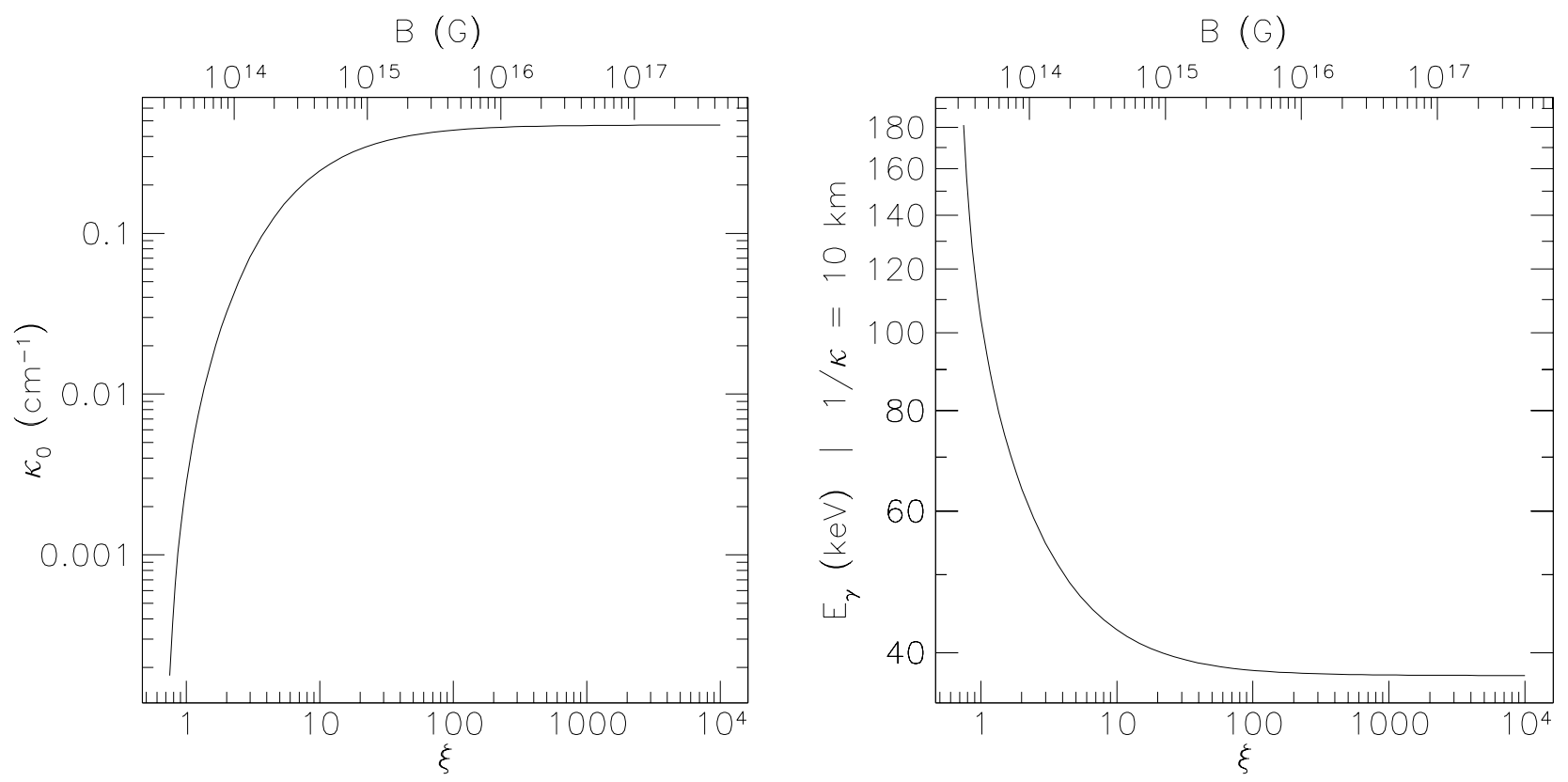

FIG. 2. The left panel depicts the photon-splitting opacity for photons with $E=m c^{2}$ as a function $\xi$ and $B$ (upper axis). The right panel shows the energy of photons with a mean-free path for splitting of ten kilometers as a function $\xi$ and $B$ 


\section{CONCLUSION}

Because of the asymptotic behavior of the function $C_{2}(\xi)$, even in immensely large fields, the photons with energies less than $37 \mathrm{keV}$ have opacities less than $(10 \mathrm{~km})^{-1}$. This energy corresponds to a temperature of $4 \times 10^{8} \mathrm{~K}$, so we must conclude that unless the strong magnetic field of the neutron star extends over a distance much greater than 10 $\mathrm{km}$, photon splitting affects the thermal radiation of only the youngest neutron stars.

We have derived a closed form expression for the Heisenberg-Euler effective Lagrangian for quantum electrodynamics as a function of the gauge and Lorentz invariant quantities $I$ and $K$ in the limit of small $K$. We have calculated from this analytic expression the photon-splitting and pair-production rates in the intense field and found them to agree with previous work. Furthermore, the expressions for the dielectric and permeability tensors in an external field derived from our analytic expression also agree with previous results [18]. We expect that these expressions may be applied to a wide variety of problems in strong electromagnetic fields, including Compton scattering, photon-photon scattering, and bremsstrahlung.

\section{ACKNOWLEDGMENTS}

This material is based upon work supported under a National Science Foundation Graduate Fellowship. L.H. thanks the National Science Foundation for support under the Presidential Faculty Fellows Program. We would also like to thank the anonymous referee for many useful comments.

[1] W. Heisenberg and H. Euler, Z. Physik 98, 714 (1936).

[2] V. S. Weisskopf, Kongelige Danske Videnskaberns Selskab, Mathematisk-Fysiske Meddelelser 14, 1 (1936).

[3] J. Schwinger, Physical Review 82, 664 (1951).

[4] W. J. Mielniczuk, J. Phys. A: Math. Gen. 15, 2905 (1982).

[5] E. W. Barnes, The Quarterly Journal of Pure and Applied Mathematics 31, 264 (1900).

[6] W. Dittrich, W. Tsai, and K.-H. Zimmermann, Phys. Rev D. 19, 2929 (1979).

[7] B. V. Ivanov, Phys. Let. B 282, 228 (1992).

[8] V. B. Berestetskii, E. M. Lifshitz, and L. P. Pitaevskii, Quantum Electrodynamics, 2nd ed. (Pergamon, Oxford, 1982).

[9] C. Itzykson and J.-B. Zuber, Quantum Field Theory (McGraw-Hill, New York, 1980).

[10] S. L. Adler, Ann. Phys. 67, 599 (1971).

[11] V. N. Baĭer, A. I. Milstein, and R. Z. Shaisultanov, hep-th/9604028 Preprint (unpublished).

[12] V. N. Bă̌er, V. M. Katkov, and V. M. Strakhovenko, Soviet Physics - JETP 40, 225 (1975).

[13] V. N. Bă̌er, V. M. Katkov, and V. M. Strakhovenko, Soviet Physics - JETP 41, 198 (1975).

[14] C. Thompson and R. C. Duncan, MNRAS 275, 255 (1995).

[15] M. G. Baring, ApJL 440, 69 (1995).

[16] S. L. Shapiro and S. A. Teukolsky, Black Holes, White Dwarfs, and Neutron Stars (Wiley-Interscience, New York, 1983).

[17] R. C. Duncan and C. Thompson, ApJL 392, 9 (1992).

[18] J. S. Heyl and L. Hernquist, in preparation. 